\title{
EDITORIAL
}

\section{Cervical cancer: Advances in prevention and knowledge of its etiology}

0 n a global basis, cervical cancer still generates close to half a million new cases per year, of which half would die as a consequence. These figures are strongly related to socioeconomic level of development, with the burden of the disease heavily affecting developing populations.

We now understand the causes of cervical cancer and novel prevention methods are being developed and introduced into medical practice. It is thus the hopeful beginning of a time that should result in the drastic reduction of cervical cancer worldwide.

Mexico cumulates risk factors for cervical cancer. The prevalence of Human Papillomavirus (HPV) DNA in women above 35 years of age is as high as $15 \%$, parity is high in most social groups and screening offers a limited coverage for a substantial proportion of the population.

In spite of efforts, the organization of screening programs such as the ones conceived and implemented in North America and in some countries in Europe has been of limited value.

For most populations it is not possible to demonstrate neither that cytology-based programs are improving nor that cervical cancer mortality is being reduced as a consequence.

This scenario is and has been dismayingly similar to other countries in the region and current estimates of the number of invasive cervical cancer still indicate some 70000 new cases per year occur in Central and South America, with a high mortality rate.

When in the early 80 's the technology was developed to allow testing for the DNA of the HPV in exfoliated cervical cells, the field exploded. With these resources, epidemiological studies were able to confirm that most of the observed risk associations were in fact mediated by the persistent presence of HPV DNA. The finding is of utmost importance because the high-risk types of HPV seem to be necessary for the development of all cervical cancer cases worldwide. As a consequence, the strategy for prevention has rapidly evolved towards the incorporation of HPV tests as a screening tool and the testing of novel HPV vaccines.

Fortunately, the number of HPV types involved in invasive cervical cancer is limited. With little geographical variation, a vaccine that would include the seven most common HPV types in cervical cancer (HPV $16,18,45,31,33,52$ and 58) would effectively prevent $87 \%$ of cases among vaccinated women. Moreover, the risk of cervical cancer for any given high-risk type of the 15 most commonly found, is not statistically different from the risk reported for the most common ones, HPV 16 and HPV 18. Therefore, under current evidence, group testing of clinical specimens for a cocktail of highrisk types should be sufficient for screening and patient management. HPV vaccines are still under evaluation, but HPV testing equipment is commercially available and FDA licensed.

A sufficient number of well-conducted studies has already been published using such cocktails and also some of the best Polymerase Chain Reaction (PCR) systems available. They have all consistently shown that any of the two, Hybrid Capture 2 (HC 2) or the GP5+/6+ PCR/EIA have sensitivities for cervical intraepithelial neoplasia 3 (CIN 3 ) and cervical cancer at least equal and in most studies significantly better than cervical cytology. Under no controlled conditions, cytology has resulted to be superior to HPV tests. In contrast, the specificity of HPV tests is age dependent. In the young age groups the specificity of the HPV tests is lower than cytology and, in the age groups 30-35 and above (again the age cutoff is country-dependent), the specificity of both tests is similar. All studies in which HC 2 or GP 5+ /6+ PCR were added to cytology as the screening method, showed that, in combination, women with both normal cytology and absence of HPV DNA have an extremely low risk of developing cervical cancer in the (predicted) 10+ subsequent years. Major gains in effectiveness and cost reduction are to be 
expected from increasing screening intervals and offering better coverage of the population at risk.

\section{What is thus ahead of us?}

Screening probably remains the only reasonable option for adult women who may never receive prophylactic vaccines on time. Next generations may witness an entirely different scenario in which adolescent vaccination may be common and cervical cancer much rarer.

However, having available an excellent novel technology is probably not enough. Vaccines or better screening tools will only work among women who receive vaccination or benefit from screening; thus issues of cost and coverage are essential. These are political and social issues, not technical anymore. Proper follow up of women at risk (either because of being carriers of high risk HPV or of advanced CIN) requires adequacy of the logistics and infrastructure, trained medical services and health education. These have all proven to be difficult in many settings in Mexico and Latin America and urban areas may preferentially receive the preventive efforts under consideration.

Advancing in cervical cancer prevention probably requires a multi-front battle to be fought. Social awareness of the problems created by cervical cancer and of the novel advances in the knowledge of its etiology is of prime importance. Empowering women and their male partners with updated health education seems an initial step that cannot be avoided. Incorporating in a reasonable manner novel technology would make screening less demanding in terms of number of visits and more efficient in terms of protection conferred by each visit. Supporting current efforts in vaccine development and testing will help in this educational respect and will train professionals and populations in the acceptance of the vaccination strategy. Adopting a scientific approach to these matters over and above vested medical interests is the crucial responsibility of the clinical and public health societies.

This monograph offers an excellent platform where to ground the effort. It includes seventeen chapters prepared by experts on the various aspects of the field, describing the issues the options and the results available. The achievement of such a task at the right time also indicates that the Mexican public health community is strongly supported by the international scientific community in the cervical cancer field and thus that future scientific support should be available when needed. It is our challenge to reduce the burden of cervical cancer mortality, an infectious disease with known and highly efficient methods of prevention. The future should be brighter than it has ever been in this respect for the developing parts of the world.

I sincerely hope you enjoy the effort

F Xavier Bosch*

\footnotetext{
* Epidemiology and Cancer Registration Unit, Catalan Institute of Oncology, Avenida Gran via s/n Km. 2.7, 08907 L'Hospitalet de Llobregat, Barcelona, Spain.
} 